Voix et Images

voixetimages

\title{
La Ville et son expression romanesque dans Bonheur d'occasion de Gabrielle Roy
}

\section{Yannick Resch}

Volume 4, numéro 2, décembre 1978

Guy Lafond

URI : https://id.erudit.org/iderudit/200155ar

DOI : https://doi.org/10.7202/200155ar

Aller au sommaire du numéro

Éditeur(s)

Les Presses de l'Université du Québec

ISSN

0318-9201 (imprimé)

1705-933X (numérique)

Découvrir la revue

\section{Citer cet article}

Resch, Y. (1978). La Ville et son expression romanesque dans Bonheur d'occasion de Gabrielle Roy. Voix et Images, 4(2), 244-257.

https://doi.org/10.7202/200155ar d'utilisation que vous pouvez consulter en ligne.

https://apropos.erudit.org/fr/usagers/politique-dutilisation/ 


\section{La Ville et son expression romanesque dans Bonheur d'occasion de Gabrielle Roy}

La relation de l'homme à son environnement, et plus précisément à la ville, est au centre des préoccupations contemporaines. II n'est que de citer les ouvrages de Pierre Sansot ${ }^{1}$, Henri Lefevre ${ }^{2}$, Pierre Medam ${ }^{3}$, Raymond Ledrut ${ }^{4}$ pour comprendre à quel point la ville, considérée comme une “poétique» ou comme l'expression d'une dialectique économique et (ou) imaginaire, pose un problème, suscite des réflexions toujours actuelles car elles mettent en jeu la vie de l'homme dans son ensemble.

Ces préoccupations se reflètent tout naturellement dans le domaine littéraire, et plus particulièrement dans les romans marqués par le phénomène de l'industrialisation. Un bon exemple en est donné par le roman québécois Bonheur d'occasion qui fait apparaître de manière très aiguë les rapports conflictuels qui pouvaient s'établir dans les années 1940 entre des individus nouvellement arrivés à la ville et l'espace urbain qui les entourait. Bonheur d'occasion, premier roman de Gabrielle Roy, paraît à Montréal en 1945 et connaît un succès reconnu en Europe puisqu'il est publié à nouveau en 1947 aux Éditions Flammarion; ce qui vaut à son auteur le prix Fémina 5 . A Montréal, le livre est salué comme un roman appartenant à une esthétique dite "réaliste ». Le directeur des Éditions Pascal, où est publié le roman, le considère comme «le premier bon roman réaliste».

Depuis, les critiques s'accordent à reconnaître que Bonheur d'occasion rompt définitivement avec la tradition terroiriste pour s'installer à la ville plus précisément dans le quartier pauvre de Saint-Henri ${ }^{6}$ ", et que par ailleurs il ouvre la voie «au roman de mœurs à incidences sociales ${ }^{7}$ ».

Cependant, si l'interaction du fait littéraire et du fait social apparaît évidente, on ne trouve guère, dans les travaux critiques consacrés à -ce roman, d'analyse précise du problème urbain et des situations dialectiques que ce problème engendre au niveau du récit.

Parmi les articles importants, on peut citer une analyse sur les personnages faite par Gérard Bessette, qui estime "qu'il ne faut pas chercher la valeur [du roman] ailleurs que dans la vie de ses personnages " et que les 
descriptions de Saint-Henri « ne suffisent pas cependant à nous donner une vue d'ensemble satisfaisante du quartier ${ }^{8}$; ; ainsi que deux études dont l'approche de type thématique et structural fait apparaître à travers les apparentes tensions du récit, l'unité de la composition'.

Mais la critique privilégiant, dans l'ensemble, l'analyse des «caractères", semble ne pas avoir vu que l'auteur avait saisi la réalité de SaintHenri moins comme un objet dont on percevait la matérialité physique que comme un organisme vivant, expression d'une réalité sociale et économique.

Je me propose donc, dans les pages qui suivent d'analyser d'abord comment les personnages perçoivent leur environnement à travers les discours qu'ils tiennent sur leur quartier considéré soit comme totalité, monde fermé sur lui-même avec ses règles, ses valeurs, ses normes; soit comme partie d'un ensemble qui constituerait le phénomène urbain et dans lequel se pose le problème de la relation d'une banlieue au centre-ville.

Ensuite, parce qu'il y a dans la ville et le phénomène urbain plusieurs systèmes de signes et de significations, je m'interrogerai sur ceux qui expriment les formes de la vie quotidienne, et cela à travers certains lieux qui mettent en évidence ce qui est vécu confusément dans le quartier.

Enfin, parce que cette réalité urbaine est œuvre de fiction, j'en dégagerai la fonction romanesque en montrant comment elle signale et signifie à la fois l'évolution des rapports qui se tissent entre les personnages.

\section{A. Caractéristiques de Saint-Henri}

\section{Un mélange de ruralité et d'urbanité}

Saint-Henri se présente, à travers les termes qui le désignent dans sa morphologie, sous une forme conflictuelle; en effet les mots les plus souvent utilisés sont: faubourg, village et quartier. C'est dire que Saint-Henri apparaît dans l'esprit de ses habitants comme un mélange de ruralité et d'urbanité.

Le mot faubourg désigne généralement un village annexé par la ville, ou la zone qui se situe à la limite de la ville. A l'inverse de la banlieue qui est une excroissance urbaine, le faubourg précède la ville et en ce sens, comme le village, il a une vie propre qui n'est pas copiée sur l'extérieur. Nous verrons que Saint-Henri possède à la fois un mode de vie et une mentalité villageoises. C'est ainsi que l'aspect rural du faubourg se lit, d'une part, dans la manière dont la nature survit un peu partout et, d'autre part, dans l'attitude et le comportement des habitants à son égard. La nature se manifeste dans les rues, les places, là où l'on l'attend le moins: "devant la petite gare, quelques fleurs poussaient leur corolle hors d'une terre galeuse». Dans la rue Saint-Ambroise, où habite Jean, il n'est resté que "deux ou trois grands arbres poussant leur racine sous le ciment du trottoir " (p. 28). 
Cette nature s'oppose à celle que l'on trouve dans les quartiers riches comme celui de Westmount où elle est protégée, recréée, soignée. Là, comme l'indique Emmanuel qui éprouve le besoin de «s'emplir les poumons d'air frais", (p. 285), on y voit des "pelouses", des «bosquets de chèvrefeuille» (285), termes qui signifient que la nature a passé par la main de l'homme. Au contraire, dans Saint-Henri la nature est «sue, entendue, vécue confusément mais globalement et sûrement s selon la formule de Pierre Sansot ${ }^{10}$. L'habitant du faubourg «ne la pose jamais devant lui comme un spectacle que l'on regarde, il la reconnaît à mille signes qui guident son comportement et son humeur". En d'autres termes, la nature n'est pas asservie aux gens, elle se développe sans ordre ni soin, témoignant d'une tenace volonté d'éclore et de pousser.

Par ailleurs, il existe dans le faubourg des séquelles de la vie paysanne: Florentine est surprise par le va-et-vient paysan qui se manifeste dans le marché et qui décrit une forme de commerce où l'individu est roi : le marchand de frites, la fruitière, la roulotte familière du marchand de tabac “un vieux de la campagne» (p. 219). Dans ce commerce, le produit vendu n'a pas été traité et s'offre dans toute sa fraîcheur, tout un vocabulaire sensoriel traduit le plaisir de la contemplation de la part de l'acheteur éventuel : pommes rondes à la peau lisse, laitue fraîche, oignon d'un bleu veiné de violet. Marché haut en couleurs et en bruits... L'auteur, évidemment, contrôle la description; il n'en reste pas moins qu'est manifesté ici de façon évidente le rapport direct, immédiat de l'homme avec le commerçant, de l'homme avec la nourriture.

Florentine se remémore ainsi son enfance et ses promenades avec son père “qui s'arrêtait pour causer avec cette forte campagnarde dont les épaules étaient recouvertes du même vieux chandail d'homme " (p. 219).

Le dimanche, Saint-Henri mène la vie paisible d'un "village dans la grande ville» : «nul quartier de Montréal [pense Emmanuel] n'a conservé ses limites précises, sa vie de village particulière, étroite, caractérisée comme Saint-Henri » (p. 252). II précise que cette vie villageoise n'est cependant visible en semaine que le soir lorsque «assis au frais sur leur pas de porte ou sur des chaises placées au bord du trottoir, ses gens s'entretiennent de seuil en seuil » (p. 253). Ce qui est indiqué ici, c'est l'absence de méflance, la solidarité qui lie entre eux les habitants d'une même rue. Plus encore, c'est une certaine cordialité qui est rendue possible par une façon de vivre le temps. Dans le faubourg on prend le temps d'échanger quelques mots avec ceux qui vivent dans le même environnement. C'est enfin ce que montre le narrateur lorsqu'il décrit Rose-Anna raccompagnant Emmanuel à la porte et "ainsi que l'on fait à la campagne elle resta un instant sur le seuil ouvert" (p. 260), pour lui adresser un dernier mot tandis que le jeune homme s'éloigne.

Cependant, si Saint-Henri par le labeur de ses habitants peut être appelé parfois une "termitière villageoise" (p. 253), ce ne peut être de la 
part de ces personnages que dans un moment d'intense émotion qui évoque un passé idyllique.

Ce qu'on voit surtout dans la morphologie de Saint-Henri c'est un présent ouvrier qui divise l'espace urbain en deux zones : les quartiers de travail et de misère dans lesquels s'insère Saint-Henri, et les quartiers résidentiels et riches qui se groupent avec la ville pour représenter dans le roman le lieu du plaisir et de la distraction. On peut retenir de ce clivage que la relation de l'habitant à son espace économique - l'opposition quartier pauvre/quartier riche est ressentie comme une opposition de classe - (mais aussi à travers un sentiment d'aliénation culturelle) - la ville, jouant un rôle ludique, apparaît comme un lieu permanent d'utopie, un lieu qui fait appel à l'imaginaire ${ }^{11}$. Je choisirai pour illustrer ces remarques l'étude d'un lieu significatif parce que traversé quotidiennement: la rue.

Si les rues Saint-Ambroise et Sainte-Zoé ont conservé un semblant d'air champêtre, les rues parcourues quotidiennement par les personnages sont sales, bruyantes, sombres et malodorantes. Saint-Henri est devenu avec l'industrialisation un quartier ouvrier où «les filatures, les élévateurs à blé ont surgi devant les maisons de bois leur dérobant la brise des espaces ouverts " (p. 28).

On peut dire, en reprenant la formule de Pierre Sansot, que le signe le plus évident de cette métamorphose est le passage d'une architecture horizontale privilégiant la petite maison individuelle à la verticalité des usines et des bâtiments de production. Quant aux rues habitées par les travailleurs et par la famille Lacasse, elles s'imposent à nous à travers un métalangage qui connote la promiscuité et la misère et que l'on peut percevoir à travers une thématique du concentré.

Dans ces rues les bruits sont plus forts qu'ailleurs, plus constants. Ils font partie du décor; que ce soit le bruit des gens : "Partout des voix aigres, des pleurs d'enfants, des cris qui jaillissaient, douloureux, des profondeurs de quelque maison, portes et volets rabattus, morte, murée sous la lumière comme une tombe" (p. 87), ou le bruit du travail et des effets de l'industrialisation du quartier: bruits de sifflets des locomotives, timbres éraillés des trams, volées de bourdons, coups de klaxons furieux, rangs d'automobiles ronronnant à l'étouffée. Cette énumération souligne non seulement la densité et la fréquence ininterrompue de ces bruits mais aussi un engorgement, un étouffement de l'espace d'où le calme et le silence sont rayés.

L'habitat subit une agression semblable : les familles s'entassent dans des pièces minuscules, la promiscuité règne dans la maison des Lacasse et les personnages sont obligés de se parler rapidement, à la dérobée sans jamais arriver à trouver le moment de calme qui permettrait une réelle communication. 
L'uniformité morne des façades que seul différencie un numéro, la saleté des trottoirs couverts de tas d'ordures, les fenêtres crasseuses aux vitres brisées, les seuils malodorants par où s'échappent les odeurs "violentes et tenaces" de linge de bébé qui n'arrive pas à sécher dans les pièces sans lumière : tout trahit le quartier misérable que la ville rejette et maintient dans sa périphérie. Le fait que les travailleurs soient refoulés du centre de la ville vers la périphérie aggrave la ségrégation géographique qui oppose le quartier limitrophe au centre ville.

\section{Relation de Saint-Henri à Montréal}

\section{La ville décrite à travers la rue Sainte-Catherine}

Nous venons de voir que l'insécurité qu'éprouvent les personnages à travers la crise du logement et la non-adaptation au quartier proviennent du fait que Saint-Henri conserve dans sa structure et son mode de vie un statut ambigu: mélange de ruralité et d'urbanité. II nous faut maintenant préciser le problème que pose la relation de Saint-Henri à Montréal ou, en d'autres termes, le rapport qui unit un quartier au centre-ville. Car la ville, dans ce roman, est essentiellement représentée par la description d'une rue : la rue Sainte-Catherine.

Cette rue s'oppose à celles de Saint-Henri que les habitants empruntent sans plaisir pour aller à leur travail et revenir chez eux. La rue SainteCatherine, c'est d'abord l'espace où l'on se promène. Pierre Sansot a montré qu'à chaque rue importante correspondait un comportement de l'usager. La marche, en effet, n'y est pas la même. On déambule dans la rue Sainte-Catherine, tandis que, la place Saint-Henri accueille un "flot las, pressé» qui marche les yeux baissés, préoccupés par des soucis ou des souffrances.

Par contre, la rue Sainte-Catherine se laisse regarder, admirer, convoiter. Jean éprouve le besoin d'y faire "une excursion hebdomadaire" pour se mêler à la foule et savourer sa puissance, car cette foule, que Florentine imagine «élégante», stimule l'imagination. Elle offre le spectacle de la beauté, de la richesse, de la liberté.

Elle est d'abord le lieu des plaisirs : distractions offertes par les restaurants et les théâtres, séduction des grands magasins aux devantures prestigieuses, fête des sens à travers les parfums des fleurs et l'éclat des lumières, prestige enfin de la personne reflétée tout au long de la promenade sur les vitres et les glaces. Bref, la rue Sainte-Catherine semble avoir concentré derrière ses vitrines tout ce qui fait appel à la détente, au plaisir, au bonheur.

Cependant, la rue Sainte-Catherine apparaît aussi comme le lieu de la plus dure réalité, car elle fait naître chez les habitants de Saint-Henri un conflit entre ce qu'ils désirent et ce qu'ils peuvent réellement acheter. II en résulte une frustration d'autant plus forte que les sollicitations et les tenta- 
tions sont nombreuses, et que le promeneur se trouve dans l'impossibilité d'y répondre. Cette relation dialectique de l'homme à la ville, Gabrielle Roy la fait apparaître très clairement dans le roman, en choisissant d'adopter le point de vue d'un de ses personnages les plus vulnérables pour décrire ce que la rue Sainte-Catherine offre aux passants. Le jeune chômeur Boisvert fait devant ses amis, chômeurs comme lui, l'inventaire de ce qui est montré et offert dans cet espace qu'est la rue Sainte-Catherine.

- Avez-vous déjà marché, vous autres, su la rue Sainte-Catherine, pas une cenne dans vot' poche, et regardé tout ce qu'y a dans les vitrines? Oui, hein! Ben moi aussi, ça m'est arrivé. Et j'ai vu du beau, mes amis, comme pas beaucoup de monde a vu du beau. Moi, j'ai eu le temps de voir du beau: pis en masse. Tout ce que j'ai vu de beau dans ma vie, à traîner la patte su la rue Sainte-Catherine, ça pourrait quasiment pas se dire! Je sais pas, moi, des Packards, des Buicks, j'en ai vu des autos faites pour le speed pis pour le fun. Pis après ça, j'ai vu leurs catins de cire, avec des belles robes de bal sur le dos, pis d'autres, qui sont habillées une miette. Qu'est-ce que vous voyezt-y pas su la rue Sainte-Catherine? Des meubles, des chambres à coucher, d'aut' catins en fanfreluches de soie. Pis des magasins de sport, des cannes de golf, des raquettes de tennis, des skis, des lignes de pêche. S'y a quelqu'un au monde qu'aurait le temps de s'amuser avec toutes ces affaires-là, c'est ben nous autres, hein?

Mais le seul fun qu'on a, c'est de les regarder. Pis la mangeaille à c'te heure! Je sais pas si vous avez déjà eu le ventre creux vous autres et que vous êtes passé par un restaurant d'iousque qu'y a des volailles qui rôtissent à petit feu sur une broche? Mais ça, c'est pas toute, mes amis. La société nous met toute sous les yeux; tout ce qu'il y a de beau sous les yeux. Mais allez pas croire qu'a fait rien que nous le mette sous les yeux! (P. 51.)

On peut noter que Boisvert qualifie ce qu'il voit de beau. Le terme est repris quatre fois; ce qui souligne l'effet esthétique du lieu sur la personne. Or ce qui est évoqué derrière cette appréciation, c'est le plaisir et le luxe.

D'abord le luxe de la toilette: «belles robes de bal ", "fanfreluches de soie" ; ces termes montrent bien que le vêtement, dans son étoffe comme dans l'usage qu'on en fait, n'a d'autre but que de servir au plaisir de la danse et des sorties, donnant ainsi l'illusion que la vie est une fête quotidienne.

Ensuite le luxe du sport individuel qui privilégie l'exploit et l'amusement personnel en supposant un niveau de vie aisé.

Enfin le confort et le plaisir résumés dans la pièce la plus symbolique pour lui : la chambre à coucher.

Par ailleurs on constate, à travers les paroles de Boisvert, que ce qui est offert dans cette rue, est le produit d'une société où règne la consommation. Aucun effort n'est demandé à celui qui achète, comme en témoignent symboliquement «les volailles qui rôtissent à petit feu ». 
Ainsi la mise en perspective du quartier Saint-Henri à travers sa place, et de Montréal par le biais de la rue Sainte-Catherine, permet à l'auteur de traduire avec évidence l'aliénation des personnages prisonniers de désirs d'autant plus violents qu'ils sont irréalisables.

Cette aliénation repose sur une ségrégation géographique qui privilégie le centre-ville par rapport au quartier et qui contribue à rendre complexe la relation de l'habitant à son environnement.

Issus du milieu paysan et de classe pauvre, venus à la ville pour survivre au moment de l'industrialisation, les travailleurs de Saint-Henri et leur famille n'arrivent pas à s'adapter au quartier qui n'est ni tout à fait un faubourg - et perd en ce sens tout ce qui en faisait un univers qui ne dépendait que du travail de la terre - ni une partie réelle de la ville puisqu'ils s'en sentent rejetés. La visite de Rose-Anna à sa mère qui la critique d'avoir épousé un citadin illustre bien cette aliénation : on sait qu'Azarius comme elle-même n'arrivent pas à s'insérer dans la réalité urbaine qui n'utilise plus d'artisans.

\section{B. Statut de quelques objets : restaurants et bars}

Ce rapport dialectique qui lie Saint-Henri à Montréal est particulièrement apparent dans Bonheur d'occasion à travers la relation des personnages principaux à certains lieux caractéristiques de la banlieue et la grande ville : les bars et les restaurants.

\section{Le restaurant du Quinze-cents}

C'est le premier espace urbain qui est donné à lire et à ce titre il joue un rôle très important. Point de départ du récit, il est aussi le premier lieu à partir duquel les autres restaurants seront mis en perspective, comparés, jugés.

Au niveau dénotatif le Quinze-cents apparaît d'abord comme un lieu où l'on étale des marchandises pour les vendre; ce qu'indiquent les termes bazar et magasin. Ensuite, il possède un foyer central : le restaurant qui occupe le fond du magasin. II faut voir dans cette localisation un aspect symbolique du lieu qui en fait un endroit protégé mais duquel on ne s'échappe guère. Ce fond est délimité par un comptoir qui révèle ce qui est confusément compris dans l'établissement, à savoir qu'il s'agit d'un lieu de passage. Cet aspect est précisé lorsque nous sont présentés les habitués du lieu : « ...travailleurs habillés de gros coutil, commis des magasins de la rue Notre-Dame [...], un chauffeur de taxi, quelques ménagères". Ces gens ne viennent pas pour se détendre ou passer un moment à discuter. Ils viennent se restaurer. Ce terme s'oppose à des mots comme manger ou déjeuner, il indique que le repas est réduit à une fonction élémentaire: celle de reprendre des forces en se restaurant rapidement d'un café bralant ou d'une assiette de frites, boisson et plat qui insistent sur le caractère illusoire et hâtif du repas. En effet, ces travailleurs se maintiennent en forme avec des excitants et des casse-croûte qui leur coupent la faim. 
Quant à l'atmosphère et au style du restaurant on peut dire qu'ils font apparaître deux systèmes de signes.

- L'un qui décrit un lieu où le travail est fatigant, parce qu'incessant et intensifié par l'exiguïté du lieu et l'engorgement des ruées de midi : le son bref et crépitant du tiroir-caisse, les bruits des vaisselles, des commandes, les "pas agités" des serveuses "se heurtant dans leur course", le déclic du grille-pain, le ronron des cafetières sur leur plaque électrique, le bourdonnement de l'appareil..., le tout formant un bruit de fond continu.

- L'autre qui décrit le restaurant comme un lieu représentatif du quartier dans sa recherche du progrès et de la modernité : “...miroitement de la verroterie des panneaux nickelés, de la ferblanterie". L'ensemble de ces termes connote un sens de tape-à-l'œil et introduit à une thématique du clinquant et du factice. On retrouve cet aspect dans le maquillage violent de Florentine et dans l'attitude des serveuses qui lisent, pense Jean, «des romans feuilletons de quinze cents et se brûlent à de paurres petits feux d'amour factice" (p. 12).

Plus encore, le restaurant est à l'image du quartier : sorte de microunivers, il résume aux yeux de Florentine «le caractère hâtif, agité et pauvre de toute sa vie passée dans Saint-Henri »(p. 9), non seulement par le cadre mais à travers les clients ennuyeux, sales et mal élevés qui se pressent, «visages ramassés sur des assiettes» (p. 17).

Enfin, ce restaurant joue sur le plan romanesque un rôle important puisqu'il est pour Florentine le seul lieu où doit s'inscrire son destin. Prisonnière de ce travail aliénant, elle compte sur les rencontres fortuites, les surprises que pourrait créer l'arrivée d'un nouveau client. Par l'intermédiaire de Jean Lévesque qui lui ouvre les portes du rêve, le restaurant a donc comme fonction de marquer les étapes d'une "éducation sentimentale» tout en laissant prévoir l'échec de la relation Jean - Florentine.

Mais ce lieu n'a d'existence significative que par ses frontières, ses limites et par ce qui le distingue d'autres lieux auxquels il peut être confronté. Bref, ce lieu n'a de sens que dans un certain rapport avec des lieux paradigmatiques d'affinité ou de dissemblance.

Cette étude rejoint ici le point de vue de Jacques Blais qui montre très clairement l'importance des «doublets et répétitions » dans Bonheur d'occasion $^{12}$.

\section{Le restaurant de la ville}

Jean invite Florentine à dîner dans un restaurant de la ville. Pour Florentine le changement est impressionnant puisqu'elle se trouve dans la situation inverse de celle qu'elle vit au "Quinze-cents" : au lieu de servir elle est servie, plutôt que de prendre des commandes elle choisit son menu. Pour que cette métamorphose soit complète Jean choisit un restaurant "en ville" et la distance qui sépare ce lieu du «Quinze-cents " est matéria- 
lisée par le tram qu'ils doivent prendre pour s'y rendre. Le restaurant participe d'une mythologie de la ville conçue comme centre de raffinement, de récréation où l'élégance et le désir de plaire sont portés à leur plus forte expression. Florentine se sent gênée d'aller dîner en ville dans sa robe de travail, et sans bijoux; sa réaction fait apparaître une mentalité "banlieusarde " immédiatement perçue par Jean qui lui se sent tout à fait à sa place (il n'a pas ressenti le besoin de changer de tenue). II éprouve de l'embarras à voir Florentine se maquiller en public et, après lui avoir conseillé de ne pas le faire à table, il dissimule mal sa déception en la voyant revenir " les lèvres épaisses de rouge et précédée d'un parfum si violent, si vulgaire que de chaque côté les consommateurs levaient la tête et souriaient" (p. 72).

Le cadre du restaurant et la carte qui est proposée font entrer Florentine dans le monde du rêve. Le décor, d'abord, exprime le raffinement et la richesse: "blancheur des nappes", «miroitement de cristaux», fleurs odorantes sur les tables. Dans ce restaurant le client fait l'objet d'une attention particulière. "Un garçon en habit noir" (p. 71) accompagne les jeunes gens à leur table et tire la chaise de Florentine.

Quant au repas, il constitue pour la jeune fille une véritable initiation au grand monde. "Tous ces mots étranges qu'elle déchiffrait syllabe par syllabe en les prononçant avec effort, la laissant désemparée, hésitante " (p. 71.)

L'embarras de Florentine fait apparaître deux éléments importants qui la classent sur le plan social. D'abord, sa méconnaissance des plats préparés et raffinés montre bien que la fonction de se nourrir ne se double pas chez elle du plaisir destiné au palais et au regard, mais qu'elle est essentiellement un moyen de remplir son estomac pour calmer sa faim. Autrement dit, manger est avant tout, pour elle, un acte vital. Ensuite, le fait qu'elle ait entre ses doigts une carte et non un menu la désoriente, car elle n'a pas l'habitude de choisir. Les clients du "Quinze-cents" prennent le menu, c'est-à-dire ce qui est meilleur marché.

II faudra donc que Jean prenne l'initiative de composer pour elle un repas. A la fin de la soirée, le fossé entre les deux personnages s'est élargi au point que Jean l'écoute à peine et la regarde avec ennui. Or, le lecteur prend conscience de cet écart à travers l'ensemble de signes qui révèlent deux comportements tout à fait différents dans un même lieu public.

Le deuxième restaurant où Jean amène Florentine, se trouve dans Saint-Henri. Ce retour à l'univers du quartier est symbolique. II indique de la part de Jean le refus de faire un effort particulier pour sortir Florentine. C'est elle, du reste, qui est venue le chercher à son travail et Jean se trouve contraint de l'inviter à dîner après avoir eu quelques paroles brusques à son égard. La description du restaurant et de l'attitude des deux jeunes gens marque une nouvelle étape dans la dégradation des rapports. Le décor 
est à peine décrit; c'est que Florentine n'éprouve plus d'émerveillement, le bâtiment n'a pas, de toute façon, le prestige de ceux de la ville. Même le repas semble ne présenter aucun intérêt, les personnages mangent peu et Jean, cette fois-ci, ne prend aucune initiative.

Au cours du repas, on a une réplique inversée de l'attitude des deux jeunes gens. Cette fois, c'est Florentine qui agit: c'est elle qui prend la main de Jean et la porte à ses lèvres tandis que celui-ci, gêné par l'arrivée du restaurateur, essaie de se dégager rapidement.

\section{Le restaurant de banlieue}

Emmanuel invite Florentine dans un restaurant qui se différencie des précédents par un décor bariolé et populaire. Situé au bord du fleuve, il a des “allures de guinguettes" et un air de fête avec ses «lampes vénitiennes" et sa "guirlande d'ampoules colorées" (p. 288).

En fait, la description met l'accent sur le mauvais goût du lieu. Tout a été sacrifié à la réclame qui recouvre les murs de la bâtisse: “C'était inimaginable tout ce que cette façade avait pu recevoir de plaques en tôle, grandes et petites, de cartons-affiches, de panneaux." (p. 288). Le jardin a un «semblant de tonnelle" et "une ombre chiche». Tous ces dètails cependant passent inaperçus aux yeux de Florentine qui trouve l'ensemble agréable et joyeux : “Ça l'air fin icitte! " Le glissement de perspective permet au lecteur de mieux cerner le caractère des personnages et en particulier de comprendre quels liens profonds lient Florentine à son quartier. Ce que Jean avait déjà remarqué est à nouveau souligné ici par l'auteur, à savoir que Florentine est beaucoup plus sensible à ce qui tape à l'œil qu'aux choses raffinées.

L'intérieur du restaurant ne fait que confirmer l'impression initiale; ici aussi l'érotisme est de mauvais aloi : «... pagodes japonaises, trirèmes en marche sur une placide mer de craie, temples hindous sur un fond crasseux $"$.

Mais ni Emmanuel ni Florentine n'y prêtent attention. Le comportement de Florentine à l'égard du repas rappelle celui qu'elle avait eu avec Jean dans le deuxième restaurant : elle mange peu, et cette indication est un signe de l'état psychologique de la jeune fille. Pendant le repas, on retrouve la même situation que lors de la première sortie de Florentine avec Jean, mais inversée. Emmanuel, à la différence de Jean, s'amuse à regarder Florentine se repoudrer et à se refaire une beauté; il la compare à un petit chat qui se débarbouille. On comprend qu'il est amoureux d'elle par le regard qu'il lui porte.

Enfin, c'est Emmanuel qui étend la main à travers la table pour prendre celle de Florentine et celle-ci, comme Jean l'avait fait, cherche à se dégager. Le lecteur voit donc clairement que si Jean fuyait Florentine, Emmanuel au contraire cherche à la conquérir. 
Ainsi, la mise en perspective de ces trois restaurants permet d'une part de dégager la réalité urbaine sous son rapport centre/périphérie, et d'autre part de suivre les étapes et l'évolution des rapports entre les personnages principaux. En effet, on a vu que ces restaurants mettent à jour des systèmes de signes qui décrivent le mode de vie des gens de Saint-Henri et de la ville à travers :

le système de l'habillement: Chez les serveurs, on notera une opposition marquée entre la tenue des serveuses du «Quinze-cents» et celle du garçon du restaurant de la ville; quant à la façon de s'habiller des clients, Florentine constate que celle de Jean diffère de celle des travailleurs du quartier.

le système du manger: On a vu, d'une part, qu'au "Quinze-cents" les clients consultaient un menu tandis qu'à la ville, les restaurants leur proposaient une carte, et d'autre part, que les desserts opposaient le "sundae spécial " aux "pâtisseries françaises ", ce qui connote l'influence française à travers une forme d'appropriation ou d'exclusion.

Par ailleurs, la description de ces restaurants permet de mettre en parallèle les couples Jean-Florentine et Emmanuel-Florentine dans des situations analogues. L'analyse de ces situations montre la dégradation progressive des rapports entre Jean et Florentine et l'acceptation par cette dernière d'un substitut de Jean: Emmanuel, qui se trouve affectivement dans la situation où Florentine se trouvait à l'égard de Jean.

\section{Les bars}

Ils jouent dans le roman un rôle complémentaire aux restaurants, car ils regroupent à l'intérieur de leurs murs une partie de la population masculine de Saint-Henri. Ces bistrots introduisent d'une manière privilégiée à la réalité de Saint-Henri. Ils vivent en effet en symbiose avec les travailleurs du quartier qui y viennent «placoter » et raconter leur malheur. Le bar de la mère Philibert, les Deux Records de Sam Latour ont ceci de particulier qu'ils sont un des rares espaces occupés dans le quartier avec plaisir. Les clients sont, ici comme là, des habitués qui retrouvent à chaque fois une virile et fraternelle amitié.

Gabrielle Roy fait apparaître, dans la description de ces lieux, une caractéristique de l'univers du faubourg et du quartier: l'importance du bistrot comme espace sécurisant et protecteur ${ }^{13}$.

On remarque dès la première description du bar de Sam Latour que ce lieu est à l'image du quartier. En effet, il tire son nom «d'un commerce fort éloigné du métier de restaurateur : la vente de disques, chansons françaises et américaines dont la vogue dépérissait à Montréal, mais qui plaisaient encore beaucoup dans Saint-Henri » (p. 36).

Le bar de Sam Latour, comme le "Quinze-cents", suit la mode de la ville mais avec un retard. 
La première fonction de ces bars est de permettre à ceux qui les fréquentent de s'exprimer, de communiquer ce qu'ils pensent, sans craindre d'être jugés ou critiqués. Dans ces lieux règnent la confiance et la camaraderie: on le notera surtout à travers l'acte de manger et de parler.

D'abord le manger: Les habitués du lieu délaissent les petites tables individuelles pour venir manger leurs hot dogs ou sandwiches au comptoir en causant avec Sam Latour. Ce qui est remarquable ici, c'est que les clients n'ont pas peur de manger devant d'autres personnes. La nourriture est simple et l'acte de se nourrir est accepté comme une des activités élémentaires de l'homme. On se rapproche du comptoir pour être plus près des autres, pour pouvoir participer au débat. Bref, l'essentiel est d'être ensemble, et l'on vient chez Sam Latour pour assurer cette solidarité. De même tous les quêteurs, chômeurs, ceux de la génération d'Emmanuel se regroupent autour de la mère Philibert, au fond de son débit.

Quant au parler, on remarque que le comportement des clients y prédispose. Sam Latour discute avec «... quatre ou cinq hommes à demi affalés sur le comptoir" (p. 37). Dans ces bistrots les gens se sentent libres dans leurs gestes, dans leurs attitudes.

La possibilité qu'ont les gens de pouvoir marcher à leur guise, la détente qui règne chez ceux qui sont assis, tout cela favorise l'art oratoire. D'une part le patron, les clients, le décor bien connu de l'orateur sont des éléments qui mettent en confiance; d'autre part, l'homme qui entre dans le bar abandonne en partie à la porte ses soucis, ses problèmes et se sent plus disponible pour discuter. C'est ainsi qu'Azarius trouve auprès des gens qui l'écoutent les mots qui lui manquent habituellement chez lui :

le mouvement oratoire lui revenait et ce sentiment agréable de porter la persuasion chez ses auditeurs; il se retourna comme pour s'adresser non pas à quelques badauds impressionnés mais à une grande foule dont la rumeur serait venue soudain remplir ses oreilles (p. 264).

Peu importe ce qui est dit, l'habitué de ces bars aime blaguer, il parle haut, avec emphase et accompagne son discours de gestes, de mimiques. Comme ce client des «Deux Records " qui «employait souvent des mots sonores qu'il déformait, dont il ne saisissait pas tout le sens mais dont il semblait écouter la résonance en lui avec un plaisir très vif " (p. 38). Les réactions sont aussi spontanées: les gens réagissent par de "gros rires" des "boutades", ou si le désaccord se fait on entend "une voix irritée" (p. 39) ou «un murmure de désapprobation". Les «Deux Records", c'est vraiment le lieu du discours pour le discours, l'endroit où l'habitué est mis en valeur, écouté comme un égal, et où, pour quelques instants, il s'élève au-dessus de cette main-d'œuvre laborieuse à laquelle il appartient et qui n'obtient jamais le droit de s'exprimer.

La deuxième fonction de ces bars, dans ce quartier voué aux déménagements perpétuels, est d'être un lieu de permanence. Emmanuel qui s'est engagé dans l'Armée éprouve le besoin, à son retour, d'aller chez la 
mère Philibert, car «marchant seul, il avait obstinément cherché des points de contact avec les habitudes d'autrefois" (p. 46). II sait que dans le débit de la grosse Emma il retrouvera la bande des jeunes chômeurs dont il partageait les jeux dans sa jeunesse.

La raison de cette permanence provient du fait que dans ces lieux les informations sur les uns et les autres sont, soit reçues, soit divulguées par un des habitués. Cela est vrai pour les situations particulières comme pour les événements politiques. C'est Emmanuel qui apporte les informations sur la guerre et ce qui se passe à l'avant, tandis que chez Sam Latour, ceux de l'arrière se groupent autour du poste de radio pour commenter les dernières nouvelles.

Quant aux problèmes personnels, il y a toujours moyen de les résoudre en parlant au comptoir. Le patron a des sources d'information qui lui permettent de trouver une solution. Ainsi Sam Latour trouve du travail pour Azarius qui vient de perdre son emploi.

On voit dans ce roman à quel point ces lieux sont révélateurs du quartier (même quand ils en sont comme l'envers) puisqu'ils offrent aux personnages un espace où ils se sentent «chez eux», et leur donne un sentiment de confiance en eux-mêmes. Ils expriment à travers le discours des habitués leurs rêves, leurs désirs mais aussi leurs préoccupations et leurs inquiétudes. Ils réalisent la seule appropriation que les travailleurs puissent avoir d'une partie de l'espace qu'ils occupent.

La description de ces lieux aide à percevoir les personnages en tant qu'individus appartenant à des groupes sociaux et éclairent les tensions dans lesquels ils sont plongés : chômage ou travail humiliant, tentation de l'engagement militaire ou planque dans le civil, etc.

Enfin ces bars, tout comme les restaurants permettent au niveau du récit de faire progresser l'action à travers la mise en parallèle des discours que tiennent les personnages à propos de la guerre, du travail ou du bonheur. C'est ainsi que le lecteur peut comparer les rapports de Jean Lévesque et d'Azarius d'une part et d'Emmanuel et d'Azarius d'autre part. Cette symétrie permet de comprendre davantage quel sera le rapport respectif de deux jeunes gens à l'égard de Florentine.

Si Bonheur d'occasion a marqué si fortement l'évolution du genre romanesque à son époque, et s'il continue à susciter de nouvelles lectures c'est, nous semble-t-il, par la question qu'il pose implicitement tout au long du récit et qui prend forme dans les thèmes comme dans la structure, à savoir comment un individu peut arriver à faire sien l'espace géographique et social dans lequel il lui est donné de vivre.

Tous les personnages peuvent se définir en fonction de l'espace, et la façon dont chacun d'eux écrit son errance dans cet enclos qu'est SaintHenri dit mieux que tout leur sentiment d'insécurité, de non-appartenance 
à un lieu qui les fait vivre en paria à la ville comme à la campagne, tout en perpétuant en eux le désir de la ville et le rêve d'un bonheur qui rappellerait la simplicité de la vie rythmée par les éléments naturels.

Yannick Resch Université d'Aix

1. Poétique de la ville, Paris, Klinsksieck, 1971.

2. Le Droit à la ville, Paris, Anthropos, 1963.

Du rural à l'urbain, Paris, Anthropos, 1970.

3. La Ville censure, Paris, Anthropos, 1971.

4. Les Images de la ville, Paris, Anthropos, 1973.

5. Nous utilisons l'édition parue chez Beauchemin, Montréal, 1970.

6. A. Vanasse, «Vers une solitude désespérante («La Notion d'étranger dans la littérature canadienne $)$, dans l'Action nationale vol. $55, n^{\circ} 7$, mars 1966 , p. 844.

7. Réjean Robidoux et André Renaud, le Roman canadien-français du xixe siècle, Ottawa, 1966, p. 75-76.

8. L'Action universitaire, t. $18, n^{\circ} 14$, juillet 1952, p. 53-74.

9. "Thèmes et structures de Bonheur d'occasion m, essai par A. Brochu dans Écrits du Canada français, t. 22, 1966, p. 163-208. L'Étude organique de Bonheur d'occasion par J. Blais dans Études litteraires, $n^{\circ} 6,1970$, Les Presses de l'Université Laval.

10. Pierre Sansot, op.cit., p. 279.

11. Pierre Medam, op. cit., p. 61-62.

12. J. Blais, op. cit., p. 28.

13. Il est intéressant de constater que la " rêverie " de Pierre Sansot sur les bistrots trouve ici sa réalisation sur le plan romanesque. 